\title{
A Distributed Navigation Strategy for Multiple Mobile Robots to Make Group Formations Adapting to Geometrical Constraints*
}

\author{
Hiroaki YAMAGUCHI** and Tamio ARAI***
}

\begin{abstract}
This paper presents a novel distributed navigation strategy of multiple holonomic mobile robots to make group formations adapting to geometrical constraints in their working environment. Each mobile robot in this navigation strategy has its own coordinate system and it senses its relative position to others, so as to make group formations. Each mobile robot especially has a two-dimensional control input referred to as a "formation vector" and the formation is controllable by the vectors. Adjusting the vectors in a distributed fashion, the group makes the formations fitting into the geometrical constraints determined by the arrangements of landmarks that specify the location of the group, and obstacles. The validity of this navigation strategy is supported by computer simulations.
\end{abstract}

Key Words: Robot, Moving Robot, Motion Control, Multi-agent Robotic System, Mobile Robot Group, Distributed Navigation, Formation Controllability, Formation Adaptation

\section{Introduction}

Multiple mobile robots form a group to cooperate with each other on accomplishing tasks that a single robot cannot handle, e.g., lunar-base construction by multiple bulldozer-type mobile robots ${ }^{(3)}$, panel construction by multiple mobile robots equipped with manipulators ${ }^{(7)}$, payload/object transportation, foraging and grazing by multiple mobile robots navigated in model-based or behavior-based control schemes ${ }^{(1),(5),(9)-(11)}$, and surveillance by multiple mobile robots with a sensor-based navigation scheme ${ }^{(13)}$. It is certainly a task for multiple robotic vehicles to make group formations, e.g., they prevent invasions in surveillance areas by making group formations adapting

* Received 16th February, 2001

** Research into Artifacts, Center for Engineering, The University of Tokyo, 4-6-1 Komaba, Meguro-ku, Tokyo 153-8904, Japan. E-mail: yamaguch@race.utokyo.ac.jp

*** Department of Systems Innovation, Faculty of Engineering, The University of Tokyo, 7-3-1 Hongo, Bunkyo-ku, Tokyo 113-8656, Japan. E-mail : arai $@$ prince.pe.u-tokyo.ac.jp to geometrical constraints in their working environment ${ }^{(14)}$, which we describe in this paper, and they capture/enclose an invader by making group formations $^{(15),(17)}$.

To make group formations, each mobile robot moves to control its relative position to others, which means that each robot has relative position feedback. Via this feedback, each robot interacts with others, so that the robots making group formations compose a large-scale multi-body system. In this paper, we especially focus on making specified formations at specified positions and, for this purpose, we put some landmarks which specify the location and the size of the final formation in their working environment where some obstacles exist. The arrangements of the landmarks and the obstacles turn into the geometrical constraints imposed on the robots in cases of making group formations. Although each robot controls its motion by itself following some rules including mathematical formulas and there is neither supervisor nor mediator, the robots make the specified formations at the specified positions in this distributed navigation strategy. Each robot specifically has its own coordinate system and it senses its relative position to the 
others, the landmarks, and the obstacles when they are visible to it. Each robot especially has a twodimensional control input referred to as a "formation vector" and the formation is controllable by the vectors. To make the specified formations at the specified positions, a robot sensing some landmarks tunes its formation vector based on some mathematical formulas designed for making the robot reach the closest landmark among them, while a robot notsensing any landmark determines its formation vector following some rules heuristically designed for each specified formation. In other words, the formation is spread by tuning the formation vectors among the landmarks whose arrangement becomes the boundary condition, and the distribution of the robots within the boundary are controlled by the formation vectors determined based on the rules heuristically designed for making each specified formation. Therefore, the formation vectors converge some values, which make the specified formation fitting into the geometrical constraint in a distributed fashion.

We show, as an example, obstructing passages by making group formations. Specifically, we suppose that a robot group makes an arc-like formation between two landmarks. A landmark is located on each side of the passage. We can put some obstacles and we can also change the relative position between the landmarks, which means we can change the geometrical constraint of the working environment of the group. However, the group adapts its formation to the variation of the constraint and it obstructs the passage by making the arc-like formation. Of course, this distributed navigation strategy is applicable under other conditions where we put more than two landmarks and where obstacles are scattered. Even if the group tries to make the other formations under the other conditions, the group adapts its formation to the geometrical constraints by tuning the formation vectors. Hence, this distributed navigation strategy is not limited in the case of making the arc-like formation obstructing the passage.

There have been significant methodologies for making group formations of multiple mobile robots $^{(4),(16)}$. Desai, Ostrowski and Kumar have presented a feedback control law used to navigate multiple nonholonomic mobile robots of the Hilaretype moving together in formations ${ }^{(4)}$. This method uses local sensor-based information in a leader-follower motion. Specifically, the leader robot is required to keep moving on a pre-planned path, and each of the remaining robots is a follower robot following the leader robot or the other robot moving ahead itself. Although all the robots have nonholonomic constraints on their mobility, moving the robots enables them to control their relative positions between them. Since the task that we consider in this paper is to make some specified formations at some positions specified by landmarks, it is not straightforward to apply this control method to our task. Yamaguchi and Burdick have shown the existence of a timevarying feedback control law that enables multiple nonholonomic mobile robots of the Hilare-type to make group formations ${ }^{(16)}$. The mobile robots are not required to keep moving and they are eventually static on making the final formation in the feedback control law. We will show in future that, based on the feedback control law, it is possible to obstruct passages by making group formations of the nonholonomic mobile robots of the Hilare-type between the two landmarks identically the same as the case of our task.

A study of self-assembling machines is another important area of research ${ }^{(12)}$. The machine consists of a number of homogeneous units, and it makes and changes its formation by controlling the connections between the units, which means that the connecting configuration is not static and it changes. On the other hand, each robot in this paper senses its relative position to other robots, which means that the robots are visually connected. In other words, the robots in the group are visually articulated. The connecting configuration of the robot group is static. The group changes its formation (which is the two-dimensional shape on the plane) by controlling the relative positions between the robots. Therefore, if the robot group can change its connecting configuration, then the formation that the group can make has more variety. This will be examined in future work.

\section{Model of Mobile Robot and Adaptation of Group Formation}

\subsection{Model of mobile robot}

We consider $(n)$ mobile robots, $(m)$ landmarks and $(l)$ obstacles on a plane. Each robot is a holonomic mobile robot free to move on a plane. We label each robot $R_{i}$, each landmark $L M_{j}$, and each obstacle $O_{j}$, but these labels are not intrinsic to this navigation strategy. Each robot does not need to know its own label, other robots', landmarks', and obstacles' labels. Especially, we denote a landmark sensed and approached by $R_{i}$ with $L M_{R_{i}}$. To make group formations at specified positions without making any collision, each robot senses its relative position to other robots, landmarks, and obstacles in its own coordinate system. We denote the coordinate system of $R_{i}$ with $\Sigma_{i}$. To express the positions of all the mobile robots in a common coordinate system, we define a static coordinate system, $\Sigma_{0}$. To express the 


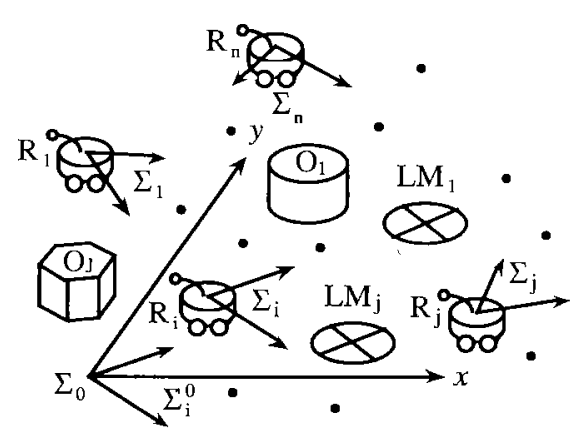

Fig. 1 Mobile robots, landmarks, and obstacles

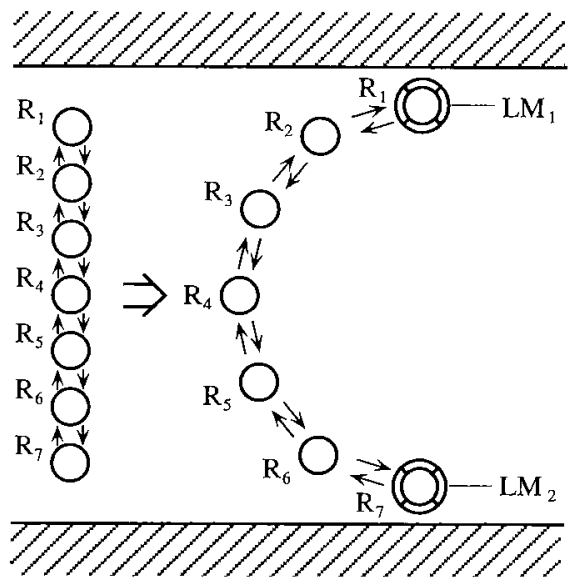

Fig. 2 Obstruction of passage by making arc-like formation

velocity of $R_{i}$, we define another coordinate system, $\Sigma_{i}^{0}$ whose origin is common to that of $\Sigma_{0}$ and whose orientation is common to that of $\Sigma_{i}$, (see Fig. 1). These coordinate systems, $\Sigma_{0}$ and $\Sigma_{2}^{0}$, are unknown to $R_{i}$. Each $R_{i}$ knows only its own coordinate system, $\sum_{i}$.

\section{2 Adaptation of group formation}

Let us consider $(n)$ mobile robots in a configuration that we refer to as an "open chain group," (see Fig. 2). An arrow in this configuration physically means that a robot at the start of the arrow is sensing its relative position to another robot at the end of the arrow, so as to make group formations. The robots in this configuration are strongly connected in a sense of graph theory. We assume that the configuration is always static. Although this navigation strategy is independent of configurations, it is practical to consider only cases of open chain groups, because robot groups easily get tangled in other configurations. As an application of this navigation strategy, we suppose that the open chain group obstructs a passage, (see Fig. 2). Specifically, we put a landmark on each side of the passage and the group makes an arc-like formation between the two landmarks. Of course, this navigation strategy is not limited in the case of making the arc-like formation and it is applicable in making other formations under other conditions where we put more than two landmarks and where obstacles are scattered. Especially, the working environment of the robots performing this task can be easily changed by making this passage wider (or narrower) or by putting some obstacles. Adjusting the formation vectors (that determine the formation and that we discuss later), the group makes its formation fit into the geometrical constraints determined by the arrangements of the landmarks and the obstacles, which we refer to as "adaptation."

Putting more landmarks is another way of controlling/making the formations. For instance, when we put an additional landmark that stands in the middle of the passage in Fig. 2 and that is apart from the line between the two landmarks, the robot group makes a "V" formation. When we put more landmarks arranged in an arc-like formation, the robot group makes the arc-like formation. In a widely open field where a closed chain group is operating, putting three landmarks around the closed chain group makes it possible for the group to have a triangle formation. Of course, the robot group is not required to have a one-dimensional configuration like an open chain group, and it is allowed to have a two-dimensional configuration like a lattice group. However, as we have described, the robot group easily gets tangled in complicated configurations, so that we consider only the cases of the open chain group in this paper.

It is possible to put the landmarks whose number is the same as that of the robots. In such cases, putting the landmarks is the same as specifying the positions of all the robots, which seems to be trivial. Therefore, we focus on the situation where the arrangement of the landmarks is the boundary condition and where the robots strive to make some specified formations within the boundary, satisfying the geometrical constraints determined by the arrangement of the landmarks and the obstacles.

The robots in this group form the one-dimensional configuration which is the open chain group, so that the initial arrangement of the robots should be one-dimensional, e.g., a line and a wide curve. Of course, we have to be, aware of that the robots fail to achieve this task in some initial arrangement. For instance, when both the end robots are arranged close to each other and the robot group has a closed chain formation initially, both the end robots approach the same landmark and the robot group fails in making the arc-like formation between the landmarks. Therefore, we have to take into account what kind of initial arrangement leads the robots to succeed in 
accomplishing this task.

\section{Robot Navigation}

\section{1 Distributed navigation strategy}

As we have described, an arrow going from a robot to another means that the robot at the start of the arrow is sensing its relative position to the other at the end of the arrow. Since the group has a strongly connected configuration, at least one arrow goes from any robot to another. We denote with $L_{i}$ the set of the robots to which arrows go from $R_{i}$. To make group formations, $R_{i}$ moves to control its relative position to the robots in $L_{i}$. Especially, we have two types of robot: one is a robot sensing some landmarks; and the other is a robot not-sensing any landmark. The robot sensing some landmarks is not required to sense all the landmarks. It senses only visible ones in the local area around itself. We give each robot some rules heuristically designed that determine whether a robot becomes a robot sensing some landmarks or a robot not-sensing any landmark. The rules are based on the configuration of the group and we describe them later. A robot sensing some landmarks tries to reach the closest landmark among them. We denote with $L M_{R_{i}}$ the landmark that $R_{i}$ senses and tries to reach. The arrangement of the landmarks are fixed in advance, and a robot group that already has a strongly connected configuration is released to make group formations fitting into the geometrical constraint determined by the arrangement of the landmarks and the obstacles. It depends on the initial locations of the robots which landmark a robot sensing some landmarks tries to reach. Each robot also controls its relative position to the other robots and the obstacles to avoid collision with them. We denote with $M_{i}$ the set of the robots sensed by $R_{i}$ for collision avoidance. The set, $M_{i}$, does not include $R_{i} \in L_{i}$. We also denote with $N_{i}$ the set of the obstacles sensed by $R_{i}$ for collision avoidance. In this distributed navigation strategy, $R_{i}$ moves at the velocity that is affected linearly by its relative position to the robots in $L_{i}$ and to $L M_{R_{i}}$ to make formations with the other robots at some position specified by the landmarks, and that is affected linearly by its relative position to the robots in $M_{i}$ and to the obstacles in $N_{i}$ for collision avoidance. The velocity of $R_{i}$ is also affected linearly by a two-dimensional control input, ${ }^{t}\left(d_{x i}^{i}, d_{y i}^{i}\right)$, referred to as a "formation vector." The upper suffix, " $i$," means that the vector is expressed in the coordinate system of $R_{i}$, i.e., in $\Sigma_{i}$. We apply this notation rule to the expression of any vector below. Formally, we express the velocity of $R_{i}$ as:

$$
\left(\begin{array}{c}
\dot{x}_{i}^{i} \\
\dot{y}_{i}^{i}
\end{array}\right)=\sum_{j \in L_{i}} \tau_{i j}\left(\begin{array}{l}
x_{j}^{i} \\
y_{j}^{i}
\end{array}\right)+\tau_{i}\left(\begin{array}{l}
x_{t i}^{i} \\
y_{t i}^{i}
\end{array}\right)+\left(\begin{array}{l}
d_{x i}^{i} \\
d_{3 i}^{2}
\end{array}\right)
$$

$$
\begin{aligned}
& \left.+\sum_{j \in L_{i} \cup M_{i} \cup N_{i}} \delta_{i j} \int\left(\begin{array}{c}
x_{j}^{i} \\
y_{j}^{i}
\end{array}\right)-D\left(\begin{array}{c}
x_{j}^{i} \\
y_{j}^{i}
\end{array}\right) /\left(\begin{array}{c}
x_{j}^{i} \\
y_{j}^{i}
\end{array}\right)\right\}, \\
\delta_{i j} & = \begin{cases}\delta & \left|t\left(x_{j}^{i}, y_{j}^{i}\right)\right| \leq D \\
0 & \left|{ }^{t}\left(x_{j}^{i}, y_{j}^{i}\right)\right|>D\end{cases}
\end{aligned}
$$

where ${ }^{t}\left(\dot{x}_{i}^{i}, \dot{y}_{i}^{i}\right)$ is the velocity of $R_{i}$ in $\sum_{i}^{0}$ whose origin is common to that of $\Sigma_{0}$ and whose orientation is common to that of $\Sigma_{i} ;{ }^{i}\left(x_{j}^{i}, y_{j}^{i}\right), j \in L_{i} \cup M_{l}$, is the position of $R_{j} \in L_{i} \cup M_{i}$ in $\Sigma_{i}$, i.e., the relative position of $R_{j} \in L_{i} \cup M_{i}$ to $R_{i}$ in $\sum_{i} ;{ }^{t}\left(x_{j}^{i}, y_{j}^{i}\right), j \in N_{i}$, is the position of a point on the surface of $O_{j} \in N_{2}$ in $\Sigma_{i}$ and the point is sensed by $R_{i}$ for collision avoidance, i.e., the relative position of the sensed point to $R_{i}$ in $\Sigma_{i}$; ${ }^{t}\left(x_{t i}^{i}, y_{t i}^{i}\right)$ is the position of $L M_{R_{i}}$ in $\Sigma_{i}$, i.e., the relative position of $L M_{R_{i}}$ to $R_{i}$ in $\sum_{i} ;{ }^{t}\left(d_{x i}^{i}, d_{y_{i}}^{i}\right)$ is the formation vector of $R_{i}$ in $\Sigma_{i} ; \tau_{i j}>0, j \in \dot{L}_{i}$, is the attraction coefficient of $R_{i}$ to $R_{j} \in L_{i} ; \tau_{i}$ is the attraction coefficient of $R_{i}$ to $L M_{R_{i}} ; \tau_{i}=\tau>0$ when $R_{i}$ senses $L M_{R_{i}}$; and $\tau_{i}=0$ when $R_{i}$ does not sense any landmark. Physically, $\tau_{i j}{ }^{t}\left(x_{i}^{i}, y_{j}^{i}\right)$ in the first term of Eq. (1) means that $R_{i}$ is attracted to $R_{j} \in L_{i}$. The second term means that $R_{i}$ is attracted to $L M_{R_{i}}$, to make the group stand at some position specified by the landmarks. The third term means that $R_{i}$ is pulled in the direction of ${ }^{t}\left(d_{x i}^{i}, d_{y i}^{i}\right)$, to make formations. The fourth term means that $R_{i}$ is repulsed from $R_{j} \in L_{i} U$ $M_{i}$ and from the surface of $O_{j} \in N_{i}$, as an artificial potential field ${ }^{(2),(6)}$ exists. This repulsion works only in cases where at least one of the relative distances of $R_{i}$ to them is shorter than $D$. The coefficient, $\delta_{i j}, j \in L_{i}$ $\cup M_{i}$, is the repulsion coefficient of $R_{i}$ from $R_{j} \in L_{i} \cup$ $M_{i} ; \delta_{i j}, j \in N_{i}$, is the repulsion coefficient of $R_{i}$ from the surface of $O_{j} \in N_{i}$. The scalar, $\delta$, is positive.

\subsection{Robotic system}

In this subsection, we give a time-differential equation describing the state variables of this whole robotic system. Specifically, to handle the positions of all the robots in a common coordinate system, we transform the vectors in Eq. (1) into vectors in Eq. (2) expressed in the static coordinate system, $\Sigma_{0}$, as:

$$
\begin{aligned}
& \left(\begin{array}{c}
\dot{x}_{z} \\
\dot{y}_{i}
\end{array}\right)=\rho_{i}\left(\begin{array}{c}
\dot{x}_{i}^{i} \\
\dot{y}_{i}^{i}
\end{array}\right) \\
& =\sum_{j \in L_{i}} \tau_{i j} \rho_{i}\left(\begin{array}{l}
x_{j}^{i} \\
y_{j}^{i}
\end{array}\right)+\tau_{i} \rho_{i}\left(\begin{array}{l}
x_{t i}^{i} \\
y_{i i}^{i}
\end{array}\right)+\rho_{i}\left(\begin{array}{l}
d_{x i}^{i} \\
d_{y i}^{i}
\end{array}\right) \\
& \left.+\sum_{j \in L_{i} \cup M_{i} \cup N_{i}} \delta_{i j} \rho_{i}\left\{\left(\begin{array}{l}
x_{j}^{i} \\
y_{j}^{i}
\end{array}\right)-D\left(\begin{array}{l}
x_{j}^{i} \\
y_{j}^{i}
\end{array}\right) / \|\left(\begin{array}{l}
x_{j}^{i} \\
y_{j}^{i}
\end{array}\right)\right)\right\} \\
& =\sum_{j \in L_{i}} \tau_{i j}\left\{\left(\begin{array}{l}
x_{j} \\
y_{j}
\end{array}\right)-\left(\begin{array}{l}
x_{i} \\
y_{i}
\end{array}\right)\right\}+\tau_{i}\left\{\left(\begin{array}{l}
x_{t i} \\
y_{t i}
\end{array}\right)-\left(\begin{array}{l}
x_{i} \\
y_{i}
\end{array}\right)\right\} \\
& +\left(\begin{array}{l}
d_{x i} \\
d_{y i}
\end{array}\right)+\sum_{j \in L_{i} \cup M_{i} \cup N_{i}} \delta_{i j}\left[\int\left(\begin{array}{l}
x_{j} \\
y_{j}
\end{array}\right)-\left(\begin{array}{c}
x_{i} \\
y_{i}
\end{array}\right)\right\} \\
& \left.\left.-D\left\{\left(\begin{array}{l}
x_{j} \\
y_{j}
\end{array}\right)-\left(\begin{array}{l}
x_{i} \\
y_{i}
\end{array}\right)\right\} /\left(\begin{array}{l}
x_{j} \\
y_{j}
\end{array}\right)-\left(\begin{array}{l}
x_{i} \\
y_{i}
\end{array}\right)\right)\right], \\
& \delta_{i j}=\left\{\begin{array}{ll}
\delta & \left|{ }^{t}\left(x_{j}, y_{j}\right)-{ }^{t}\left(x_{i}, y_{i}\right)\right| \leq D \\
0 & \left|{ }^{t}\left(x_{j}, y_{j}\right)-{ }^{t}\left(x_{i}, y_{i}\right)\right|>D
\end{array},\right.
\end{aligned}
$$

where ${ }^{t}\left(\dot{x}_{i}, \dot{y}_{i}\right)$ is the velocity of $R_{i}$ in $\Sigma_{0} ; \rho_{i}$ is the 
transformation matrix from $\sum_{i}^{0}$ to $\Sigma_{0} ;{ }^{t}\left(x_{j}, y_{j}\right), j \in L_{i}$ $\cup M_{i}$, is the position of $R_{j} \in L_{i} \cup M_{i}$ in $\Sigma_{0} ;{ }^{t}\left(x_{i}, y_{i}\right)$ is the position of $R_{i}$ in $\Sigma_{0} ;{ }^{t}\left(x_{j}, y_{j}\right), j \in N_{i}$, is the position of a point on the surface of $O_{j}, j \in N_{i}$, in $\Sigma_{0}$ and it is sensed by $R_{i}$ for collision avoidance ; ${ }^{t}\left(x_{t i}, y_{t i}\right)$ is the position of $L M_{R_{i}}$ sensed and approached by $R_{i}$ in $\Sigma_{0}$; and ${ }^{t}\left(d_{x i}, d_{y i}\right)$ is ${ }^{t}\left(d_{x i}^{i}, d_{y i}^{i}\right)$ transformed by $\rho_{i}$ and it is the formation vector of $R_{i}$ expressed in $\Sigma_{0}$. We rewrite Eq. (2) as :

$$
\begin{aligned}
& \left(\begin{array}{l}
\dot{\boldsymbol{x}} \\
\dot{\boldsymbol{y}}
\end{array}\right)=\left(\begin{array}{cc}
B+C-\Delta-\Lambda & 0 \\
0 & B+C-\Delta-\Lambda
\end{array}\right)\left(\begin{array}{l}
\boldsymbol{x} \\
\boldsymbol{y}
\end{array}\right) \\
& +\left(\begin{array}{cc}
\Delta & 0 \\
0 & \Delta
\end{array}\right)\left(\begin{array}{l}
\boldsymbol{x}_{t} \\
\boldsymbol{y}_{t}
\end{array}\right)+\left(\begin{array}{l}
\boldsymbol{d}_{x} \\
\boldsymbol{d}_{y}
\end{array}\right)+\left(\begin{array}{l}
\boldsymbol{X} \\
\boldsymbol{Y}
\end{array}\right) \\
& \boldsymbol{x}={ }^{t}\left(x_{1}, x_{2}, \cdots, x_{n}\right), \boldsymbol{y}={ }^{t}\left(y_{1}, y_{2}, \cdots, y_{n}\right) \text {, } \\
& \boldsymbol{x}_{t}={ }^{t}\left(x_{t 1}, x_{t 2}, \cdots, x_{t n}\right), \boldsymbol{y}_{t}={ }^{t}\left(y_{t 1}, y_{t 2}, \cdots, y_{t n}\right) \text {, } \\
& \boldsymbol{d}_{x}={ }^{t}\left(d_{x 1}, d_{x 2}, \cdots, d_{x n}\right), \boldsymbol{d}_{y}={ }^{t}\left(d_{y 1}, d_{y 2}, \cdots, d_{y n}\right) \text {, } \\
& \Delta=\operatorname{diag}\left(\tau_{1}, \tau_{2}, \cdots, \tau_{n}\right) \text {, } \\
& \Lambda=\operatorname{diag}\left(\sum_{j \subseteq N_{1}} \delta_{1 j}, \sum_{j \in N_{2}} \delta_{2 j}, \cdots, \sum_{j \in N_{n}} \delta_{n j}\right) \text {, } \\
& \boldsymbol{X}={ }^{t}\left(X_{1}, X_{2}, \cdots, X_{n}\right), \boldsymbol{Y}={ }^{t}\left(Y_{1}, Y_{2}, \cdots, Y_{n}\right) \text {, } \\
& X_{i}=\sum_{j \in N_{i}} \delta_{i j} x_{j}-\sum_{j \in L_{i} \cup M_{i} \cup N_{i}} \delta_{i j} D\left(x_{j}-x_{i}\right) /\left.\right|^{t}\left(x_{j}, y_{j}\right) \\
& -{ }^{t}\left(x_{i}, y_{i}\right) \mid \\
& Y_{i}=\sum_{j \in N_{i}} \delta_{i j} y_{j}-\sum_{j \in L_{i} \cup M_{i} \cup N_{2}} \delta_{i j} D\left(y_{j}-y_{i}\right) /\left.\right|^{t}\left(x_{j}, y_{j}\right) \\
& { }^{t}\left(x_{i}, y_{i}\right) \mid \text {, }
\end{aligned}
$$

where $B, C \in \boldsymbol{R}^{n \times n}$. The components of $B$ are given as : $b_{i j, i \neq j}=\tau_{i, j \in L_{2}}>0, b_{i j, i \neq j}=\tau_{i j \notin L_{i}}=0$, and $b_{i j}=-\sum_{, j=1, j \neq i}^{n} b_{i j}$. The components of $C$ are given as: $c_{i j, i \neq j}=\delta_{i j \in L_{t} \cup M_{i}}, c_{i j, i \neq j}=\delta_{i j \notin L_{i} \cup M_{i}}=0$, and $c_{i i}=$ $-\sum_{j=1, j \neq i}^{n} c_{i j}$. The set, $L_{i}$, does not include $R_{j} \in M_{i}$. As we have described, a group has a strongly connected configuration, so that $b_{i i}<0, i=1,2, \cdots, n$ Since the diagonal components of $B$ are negative and the nondiagonal ones are not negative, $B$ is a compartment matrix. The matrix, $C$, is also a compartment matrix. Using Geršgorin's and Perron-Frobenius theorems $^{(8)}$, we can easily prove that all the eigenvalues of $B$ are asymptotically stable except one and only one zero-eigenvalue whose eigenvector is parallel to $\boldsymbol{v}_{1}={ }^{t}(1,1, \cdots, 1) \in \operatorname{Ker}(B)$. Similarly, all the eigenvalues of $C$ are also asymptotically stable except one and only one zero-eigenvalue whose eigenvector is also parallel to $v_{1} \in \operatorname{Ker}(\mathrm{C})$. Then, it follows from $(B+C-\Delta-\Lambda) v_{1} \leq 0$ that $(B+C-\Delta$ $-A$ ) is asymptotically stable(8). In Eq. $(3)$, the norms of $\boldsymbol{X}$ and $\boldsymbol{Y}$ are finite, because ${ }^{t}\left(X_{i}, Y_{i}\right)$ is the summation of two vectors: one is the summation of $\delta_{i j}{ }^{t}\left(x_{j}\right.$, $\left.y_{j}\right), j \in N_{i}$, where ${ }^{t}\left(x_{j}, y_{j}\right), j \in N_{i}$, is the position of a point on the surface of $O_{j} \in N_{i}$ in $\Sigma_{0}$ and the point is sensed by $R_{i}$ for collision avoidance; and the other is the summation of the normalized vectors, and the norm of each vector is $\delta_{i j} D$, (see Eq. ( 3$)$ ). Therefore, if we design the norm of ${ }^{t}\left(d_{x 2}, d_{y i}\right)$ to be finite, then $x$ and $\boldsymbol{y}$ are stable and they are determined by the formation vectors and the locations of the landmarks and the obstacles. In the following, we describe that the formation is spread among the landmarks (whose arrangement is the boundary condition on making formations) by tuning the formation vectors of the robots sensing some landmarks.

\section{3 Tuning formation vectors}

In this navigation strategy, we suppose that a group spreads its formation among the landmarks whose arrangement is the boundary condition. For instance, a landmark is located on each side of a passage and an open chain group spreads its arc-like formation between the two landmarks, (see Fig. 2). To make this possible, we propose a distributed tuning method for the formation vector of a robot that senses some landmarks and that tries to reach the closest landmark, $L M_{R_{i}}$, among them. As Eq. (1) shows, $R_{i}$ is attracted to the robots in $L_{i}$ and it is also attracted to the closest landmark, $L M_{R_{i}}$, when $R_{i}$ is a robot sensing some landmarks. Of course, the robot sensing some landmarks is not required to sense all the landmarks. It senses only visible ones in the local area around itself. In addition, $R_{i}$ is pulled in the direction of its formation vector, and it is repulsed from the robots in $M_{i}$ and from the obstacles in $N_{i}$ for collision avoidance. In order to reach $L M_{R_{i}}, R_{i}$ needs to make these forces (that are attractive, pulling and repulsive forces and that drive $R_{i}$ ) be in balance on standing in the location of $L M_{R_{i}}$, otherwise $R_{i}$ stops on its way to $L M_{R_{i}}$. For this purpose, we propose that $R_{i}$ tunes its formation vector as:

$$
\left(\begin{array}{c}
\dot{d}_{x i}^{2} \\
\dot{d}_{y i}^{i}
\end{array}\right)=-\sum_{j \in L_{i}} \tau_{i j}\left(\begin{array}{l}
x_{j}^{i} \\
y_{j}^{i}
\end{array}\right)-\left(\begin{array}{l}
d_{x i}^{i} \\
d_{y_{i}}^{i}
\end{array}\right)
$$

The equation, Eq. (4), physically means that ${ }^{t}\left(d_{x i}^{i}, d_{y i}^{i}\right)$ converges as:

$$
\left(\begin{array}{l}
d_{x i}^{i} \\
d_{y i}^{i}
\end{array}\right)=-\sum_{j \in L_{i}} \tau_{i j}\left(\begin{array}{l}
x_{j}^{i} \\
y_{j}^{i}
\end{array}\right) .
$$

Substituting the right hand side of Eq. (5) for ${ }^{t}\left(d_{x i}^{i}, d_{y i}^{i}\right)$ in the right hand side of Eq. $(1)$, we derive

$$
\begin{aligned}
\left(\begin{array}{c}
\dot{x}_{i}^{i} \\
\dot{y}_{i}^{i}
\end{array}\right) & =\tau_{i}\left(\begin{array}{l}
x_{t i}^{i} \\
y_{t i}^{i}
\end{array}\right) \\
& \left.+\sum_{j \in L_{i} \cup M_{i} \cup N_{i}} \delta_{i j}\left[\left(\begin{array}{c}
x_{j}^{i} \\
y_{j}^{i}
\end{array}\right)-D\left(\begin{array}{l}
x_{j}^{i} \\
y_{j}^{i}
\end{array}\right)\right) /\left(\begin{array}{l}
x_{j}^{i} \\
y_{j}^{i}
\end{array}\right)\right\}, \\
\delta_{i j} & = \begin{cases}\delta & \left|t\left(x_{j}^{i}, y_{j}^{i}\right)\right| \leq D \\
0 & \left|t\left(x_{j}^{i}, y_{j}^{i}\right)\right|>D\end{cases}
\end{aligned}
$$

The first term in the right hand side of Eq. (6) means that $R_{i}$ is attracted to $L M_{R_{i}}$ and the second term means that $R_{i}$ is repulsed from the robots in $M_{i}$ and from the obstacle in $O_{j}$ for collision avoidance. Since the repulsion works only the limited areas around the robots and the obstacles, $R_{i}$ moves toward $L M_{R_{i}}$ and approaches it. In addition, each landmark is a two ${ }^{-}$ dimensional circle-shape mark on the plane and it has an enough space open to accommodate a single robot, so that there is no obstacle repulsing the robot on the landmark whose center is the final destination of the 
robot, (see Fig. 2). Of course, the robot approaching the landmark cannot reach it, when a robot in another group already stands on the landmark. This is a confliction between them. We will examine to solve such confliction in a distributed fashion in future work. If the repulsion does not make any deadlock on the way of $R_{i}$ to $L M_{R_{i}}$, we can neglect the second term in Eq. (6) as :

$$
\left(\begin{array}{c}
\dot{x}_{i}^{i} \\
\dot{y}_{i}^{i}
\end{array}\right)=\tau_{i}\left(\begin{array}{c}
x_{t i}^{i} \\
y_{t i}^{i}
\end{array}\right) \text {. }
$$

To describe the physical meaning of Eq. (7), we transform all the vectors in Eq. ( 7 ) into vectors in the static coordinate system, $\Sigma_{0}$, as :

$$
\begin{gathered}
\left(\begin{array}{c}
\dot{x}_{i} \\
\dot{y}_{i}
\end{array}\right)=\rho_{i}\left(\begin{array}{c}
\dot{x}_{i}^{i} \\
\dot{y}_{i}^{i}
\end{array}\right)=\tau_{i} \rho_{i}\left(\begin{array}{c}
x_{t i}^{i} \\
y_{t i}^{i}
\end{array}\right) \\
=\tau_{i}\left\{\left(\begin{array}{c}
x_{t i} \\
y_{t i}
\end{array}\right)-\left(\begin{array}{c}
x_{i} \\
y_{i}
\end{array}\right)\right\} .
\end{gathered}
$$

The equation, Eq. ( 8 ), physically means that $R_{i}$ moves toward $L M_{R_{i}}$ and finally reaches it. Therefore, if there is no deadlock on the way of $R_{i}$ to $L M_{R_{i}}, R_{i}$ can reach $L M_{R_{i}}$, tuning its formation vector by Eq. (4). Since the right hand side of Eq. (4) consists of the relative position of $R_{i}$ to the robots in $L_{i}$ and its formation vector, each $R_{i}$ can execute Eq. (4) independently.

\section{4 Determination of formation vectors}

We have two types of robot in this navigation strategy. One is a robot sensing some landmarks and it tries to reach the closest landmark, $L M_{R i}$, among them, tuning its formation vector by Eq. (4). The other is a robot not-sensing any landmark. Thus, the arrangement of the landmarks becomes the boundary condition. The distribution of the robots not-sensing any landmark within the boundary is also controlled by their formation vectors. It depends on some rules that are designed and given to the robots and that are based on the configuration of the group whether $R_{i}$ becomes a robot sensing some landmarks or a robot not-sensing any landmark. We describe the rules in simulations later. In this navigation strategy, we suppose that $R_{i}$ not-sensing any landmark determines its formation vector, ${ }^{t}\left(d_{x i}^{i}, d_{y i}^{i}\right)$, in its coordinate system, $\Sigma_{i}$, solely according to the formation vectors of the robots in $L_{i}$ that are sensed by $R_{z}$ so as to make group formations with other robots. In this subsection, we specifically give two examples of the determination of the formation vector, and they are applied to the case of making an arc-like formation obstructing a passage, (see Fig. 2). Of course, this navigation strategy is not limited in such case and it is possible to make other formations under other conditions where we put more than two landmarks and where obstacles are scattered.

We show a determination of the formation vector

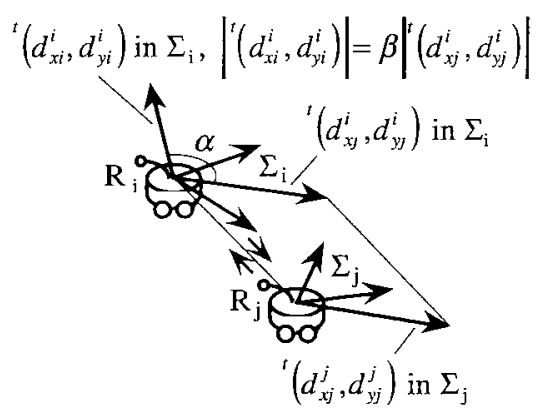

Fig. 3 Determination of ${ }^{t}\left(d_{x i}^{i}, d_{y i}^{i}\right)$

of $R_{i}$ not-sensing any landmark in Fig. 3. The robot, $R_{i}$, determines its formation vector, ${ }^{t}\left(d_{x i}^{i}, d_{y i}^{i}\right)$, to be the vector whose orientation is that of ${ }^{t}\left(d_{x j}^{i}, d_{y_{j}}^{i}\right)$ rotat ed by $\alpha$, i.e.,

$$
\left(\begin{array}{l}
d_{x i}^{i} \\
d_{y i}^{i}
\end{array}\right) /\left|\left(\begin{array}{l}
d_{x i}^{i} \\
d_{y i}^{i}
\end{array}\right)\right|=\left(\begin{array}{cc}
\cos \alpha & -\sin \alpha \\
\sin \alpha & \cos \alpha
\end{array}\right)\left(\begin{array}{l}
d_{x j}^{i} \\
d_{y j}^{i}
\end{array}\right) /\left|\left(\begin{array}{l}
d_{x j}^{i} \\
d_{y j}^{i}
\end{array}\right)\right|
$$

and whose norm is $\beta^{t}\left(d_{x j}^{i}, d_{y j}^{i}\right) \mid$, i.e.,

$$
\left|\left(\begin{array}{l}
d_{x i}^{i} \\
d_{y_{i}}^{i}
\end{array}\right)=\beta\right|\left(\begin{array}{l}
d_{x j}^{i} \\
d_{y_{j}}^{i}
\end{array}\right) \mid
$$

The vector, ${ }^{t}\left(d_{x j}^{i}, d_{y_{j}}^{i}\right)$, is the formation vector of $R_{j}$ expressed in $\Sigma_{i}$, which means that $R_{j}$ informs $R_{i}$ of its formation vector expressed in $\Sigma_{j},{ }^{t}\left(d_{x j}^{j}, d_{y j}^{j}\right)$, by communicating with $R_{i}$, and which also means that $R_{i}$ transforms the vector into ${ }^{t}\left(d_{x j}^{i}, d_{y j}^{i}\right)$ by the transformation matrix from $\Sigma_{j}$ to $\Sigma_{i}$. To perform this transformation, $R_{i}$ is required to know the relative orientation of $\Sigma_{j}$ with respect to $\Sigma_{i}$. Since both $R_{i}$ and $R_{j}$ sense the relative position between them, $R_{i}$ senses the relative position of $R_{j}$ to $R_{i}$ expressed in $\Sigma_{i}$. In addition, $R_{j}$ informs $R_{i}$ of the relative position of $R_{i}$ to $R_{j}$ expressed in $\Sigma_{j}$ by communicating with $R_{i}$. Both the relative positions: one is expressed in $\Sigma_{i}$; and the other is expressed in $\Sigma_{j}$ are uniquely associated with each other by the transformation matrix that is a rotation matrix, so that $R_{i}$ can calculate the relative orientation of $\Sigma_{j}$ with respect to $\Sigma_{i}$. In other words, we are assuming the communication between $R_{i}$ and $R_{j}$. Such communication generally has delay and errors, so that the transformation is not accurate and such inaccuracy deforms the final formation. The magnitude of the deformation is proportional to the inaccuracy.

We show another determination of the formation vector of $R_{i}$ not-sensing any landmark as :

$$
\left(\begin{array}{l}
\dot{d}_{x i}^{i} \\
\dot{d}_{y i}^{i}
\end{array}\right)=\sum_{j \in L_{i}}\left\{\left(\begin{array}{l}
d_{x j}^{i} \\
d_{y j}^{i}
\end{array}\right)-\left(\begin{array}{l}
d_{x i}^{i} \\
d_{y i}^{i}
\end{array}\right)\right\}
$$

This equation physically means that $R_{i}$ makes its formation vector, ${ }^{t}\left(d_{x i}^{i}, d_{y i}^{i}\right)$, converge to the average of the formation vectors among the robots in $L_{i}$. In this case, $R_{i}$ knows ${ }^{t}\left(d_{x j}^{j}, d_{y, j}^{j}\right)$ and $R_{i}$ calculates the relative orientation of $\Sigma_{j}$ with respect to $\Sigma_{i}$ by communicating with $R_{j}$ similarly as we have described in 


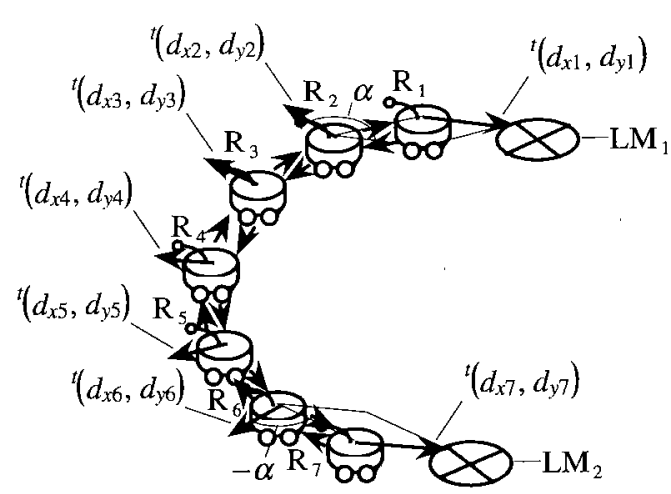

Fig. 4 A mobile robot group

Fig. 3.

\section{Simulations}

We simulated this navigation strategy as shown in Fig. 4 where seven holonomic mobile robots in an open chain group try to make an arc-like formation between two landmarks. To make such formation, we give some rules to the robots in advance as: i ) only an end robot senses some landmarks when they are visible in the local area around itself and it tries to reach the closest landmarks among them, tuning its formation vector by Eq. (4); ii) a non-end robot determines its formation vector as shown in Fig. 3 when it is a neighbor of an end robot; and iii) a nonend robot determines its formation vector by Eq. (9) when it is not a neighbor of an end robot. Since an end robot senses only one robot while a non-end robot senses two robots, each robot can recognize whether it is an end or not. We refer to a robot as a "neighbor of an end robot" when the robot and the end robot are sensing the relative position between them, i.e., they have two arrows between them. The robots, $R_{1}$ and $R_{7}$, are the end robots in Fig. 4 and they tune their formation vectors by Eq. (4). The others are the non-end robots. The robots, $R_{2}$ and $R_{6}$, are the neighbors of the end robots and they determine their formation vectors as shown in Fig. 3 . The robots, $R_{3}$, $R_{4}$ and $R_{5}$, are not the neighbors of the end robots and they determine their formation vectors by Eq. (9). The rotating angle for the formation vector of $R_{2}$ is $\alpha$ while that of $R_{6}$ is $-\alpha$, (see Fig. 4). The landmark, $L M_{1}$, has a plus sign, $(+)$, while the other landmark, $L M_{2}$, has a minus sign, (-). The plus sign is transmitted from $L M_{1}$ to $R_{2}$ through $R_{1}$ and the minus sign is transmitted from $L M_{2}$ to $R_{6}$ through $R_{7}$. Thus, we are assuming communication that enables the robots to know whether they are the neighbors of the end robots or not, and that also enables the robots to know the signs transmitted from the landmarks. We set the attraction coefficient of $R_{i}$ to $R_{j}$ as : $\tau_{i j}=\tau_{j i}=$ $20.0, j=i+1, i=1,2, \cdots, 6$. We also set the parameters

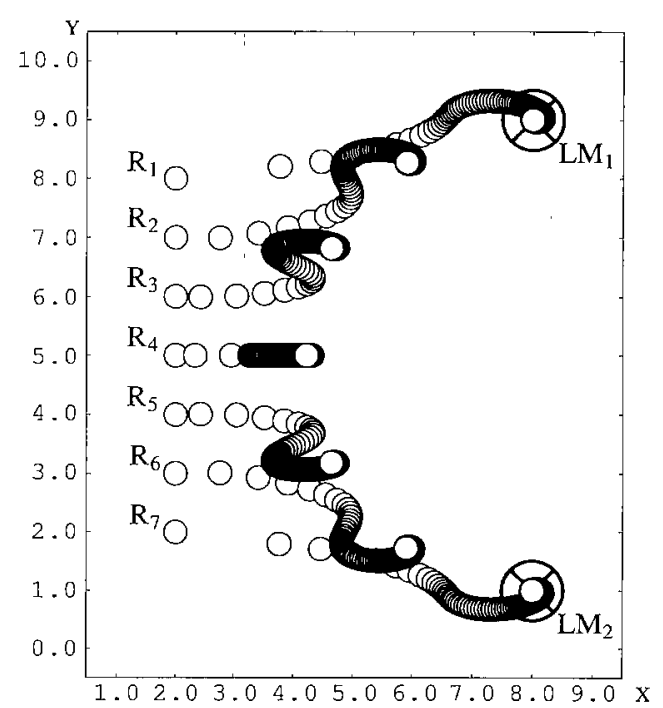

Fig. 5 A simulation result

as : $\tau=5.0, \delta=150.0, D=1.0, \alpha=2.0 \pi / 3.0[\mathrm{rad}]$, and $\beta$ $=0.5$.

Figure 5 shows a simulation result. We put $L M_{1}$ and $L M_{2}$ on the coordinates, $(8.0,9.0)$ and $(8.0,1.0)$, respectively. All the robots are initially located on the line, $x=2.0$, at an interval of 1.0 as:

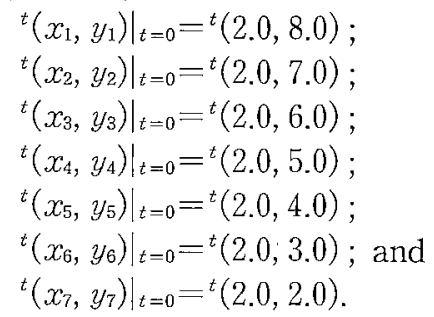

The formation vectors are initially set as :

$$
\begin{aligned}
& \left.{ }^{t}\left(d_{x 1}, d_{y 1}\right)\right|_{t=0}={ }^{t}(0.0,20.0) ; \\
& \left.{ }^{t}\left(d_{x 7}, d_{y 7}\right)\right|_{t=0}={ }^{t}(0.0,-20.0) ; \text { and } \\
& \left.{ }^{t}\left(d_{x i}, d_{y i}\right)\right|_{t=0}={ }^{t}(0.0,0.0), \\
& i=3,4,5 .
\end{aligned}
$$

Since $R_{2}$ and $R_{6}$ determine their formation vectors as shown in Fig. 4, the initial values of these vectors are given as :

$$
\begin{aligned}
& \left.{ }^{t}\left(d_{x 2}, d_{y 2}\right)\right|_{t=0}={ }^{t}(-17.3,-10.0) ; \text { and } \\
& \left.{ }^{t}\left(d_{x 6}, d_{y 6}\right)\right|_{t=0}={ }^{t}(-17.3,10.0) .
\end{aligned}
$$

We can see that $R_{1}$ and $R_{7}$ finally reach $L M_{1}$ and $L M_{2}$, respectively, tuning their formation vectors by Eq. (4), and the robot group makes an arc-like formation between the two landmarks, to obstruct a passage.

Figure 6 shows another simulation result. We put $L M_{1}$ and $L M_{2}$ on the coordinates, $(8.0,8.0)$ and (5.0, 2.0 ), respectively. The other conditions remain the same as those of Fig. 5. We can see that the robot group makes an arc-like formation between the two landmarks. In other words, the robot group adapts its formation to the variation of the relative position between the landmarks. The final formation in Fig. 6 


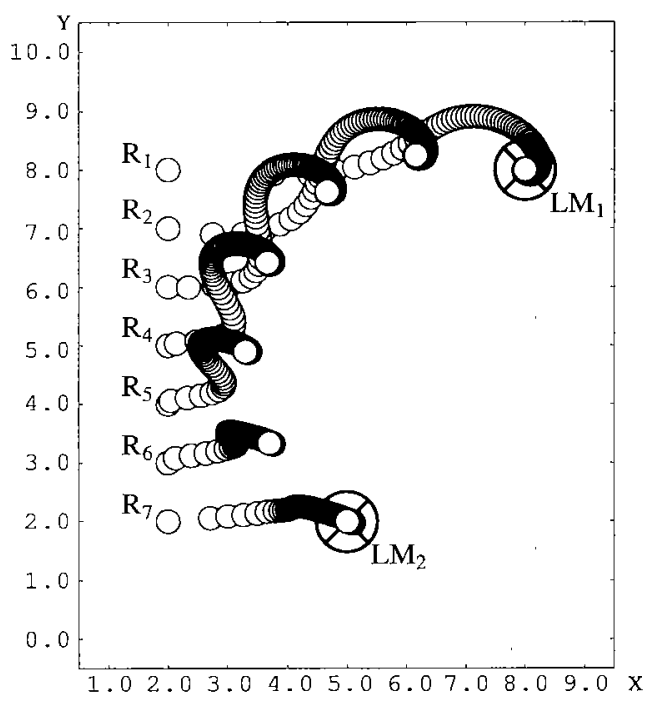

Fig. 6 A simulation result

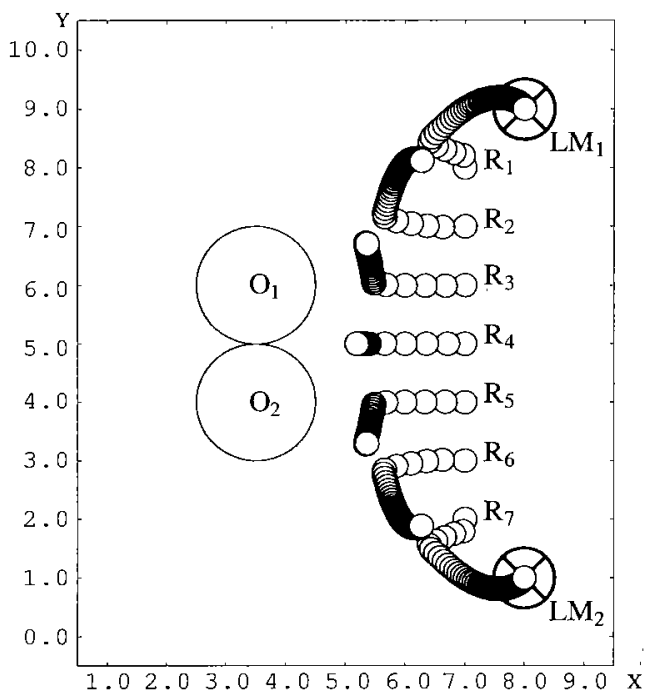

Fig. 7 A simulation result

has a similar figure of the final formation in Fig. 5 while its size is smaller, because the formation is linearly controlled by the formation vectors.

Figure 7 shows another simulation result. We put two cylinders (obstacles). The radius of each one is 1.0 , and their centers are located on the coordinates, $(3.5,4.0)$ and $(3.5,6.0)$, respectively. All the robots are initially located on the line, $x=7.0$ at an interval of 1.0 as:

$$
\begin{aligned}
& \left.{ }^{t}\left(x_{1}, y_{1}\right)\right|_{t=0}={ }^{t}(7.0,8.0) ; \\
& \left.{ }^{t}\left(x_{2}, y_{2}\right)\right|_{t=0}={ }^{t}(7.0,7.0) ; \\
& \left.{ }^{t}\left(x_{3}, y_{3}\right)\right|_{t=0}={ }^{t}(7.0,6.0) ; \\
& \left.{ }^{t}\left(x_{4}, y_{4}\right)\right|_{t=0}={ }^{t}(7.0,5.0) ; \\
& \left.{ }^{t}\left(x_{5}, y_{5}\right)\right|_{t=0}={ }^{t}(7.0,4.0) ; \\
& \left.{ }^{t}\left(x_{6}, y_{6}\right)\right|_{t=0}={ }^{t}(7.0,3.0) ; \text { and } \\
& \left.{ }^{t}\left(x_{7}, y_{7}\right)\right|_{t=0}={ }^{t}(7.0,2.0)
\end{aligned}
$$

The other conditions remain the same as those of Fig. 5. We can see that the robot group makes an arc-like formation that is deformed by the repulsion from the obstacles between the two landmarks. In other words, the robot group adapts its formation to the geometrical constraint of the arrangement of the landmarks and the obstacles. If the robots have the initial positions of Fig. 5 and we, put an obstacle between the initial position of $R_{4}$ and the center of the two landmarks, then the robot group is trapped by the obstacle and it makes a $V$-formation. It is possible for the robot group to avoid being trapped by cutting and reconnecting the visual connection (attraction) between the robots, which will be examined in future work.

\section{Conclusions}

This paper has presented a novel distributed navigation strategy that enables multiple holonomic mobile robots to make group formations, and that also enables them to make the group formations fit into geometrical constraints in their working environment, which we refer to as "adaptation." Each robot in this navigation strategy has its own coordinate system, and it controls its relative position to others, landmarks, and obstacles, so as to make the group formations at some positions specified by the land marks. In other words, the arrangement of the landmarks becomes the boundary condition, and the robots make the group formations within the boundary. Each robot especially has a two-dimensional control input referred to as a "formation vector" and the group formation is controllable by the vectors. Adjusting the formation vectors in a distributed fashion, the robot group makes its formation fit into the geometrical constraints determined by the arrangements of the landmarks and the obstacles. We have shown, as an example, that the robot group obstructs a passage by making an arc-like formation between the two landmarks and we have also shown that the robot group adapts its formation to the variation of such constraints. The validity of this navigation strategy is supported by computer simulations.

\section{References}

(1) Arai, T. and Ota, J., Dwarf Intelligence-A Large Object Carried by Seven Dwarves-, Journal of Robotics and Autonomous Systems, Vol. 18 (1996), pp. 149-155.

(2) Borenstein, J. and Koren, Y., Real-time Obstacle Avoidance for Fast Mobile Robots, IEEE Transactions on Robotics and Automation, Vol.19, No. 5 (1989), pp. 1179-1187.

(3) Brooks, R.A., Maes, P., Mataric, M.J. and More, G., Lunar Base Construction Robots, Proceedings of the 1990 IEEE/RSJ International Workshop on Intelligent Robots and Systems (IROS '90), Tsuchiura, Tsukuba, Japan, (1990), pp. 389-392. 
(4) Desai, J.P., Ostrowski, J. and Kumar, V., Controlling Formations of Multiple Mobile Robots, Proceedings of the 1998 IEEE International Conference on Robotics and Automation (ICRA '98), Leuven, Belgium, (1998), pp. 2864-2869.

(5) Johnson, P.J. and Bay, J.S., Distributed Control of Simulated Autonomous Mobile Robot Collectives in Payload Transportation, Autonomous Robots, Vol. 2, No. 1 (1995), pp. 43-63.

(6) Khatib, O., Real-time Obstacle Avoidance for Manipulators and Mobile Robots, the International Journal of Robotics Research, Vol. 5, No. 1 (1980), pp. 90-98.

(7) Khatib, O., Yokoi, K., Chang, K., Ruspini, D., Holmberg, R. and Casal, A., Coordination and Decentralized Cooperation of Multiple Mobile Manipulators, Journal of Robotic Systems, Vol. 13, No. 11 (1996), pp. 755-764.

(8) Kodama, S. and Suda, N., Matrix Theory for System Control, Society of Instrument and Control Engineers (SICE in Japanese), (1978).

(9) Kube, C.R. and Zhang, H., Task Modeling in Collective Robotics, Autonomous Robots, Vol. 4, No. 1 (1997), pp. 53-72.

(10) Mataric, M.J., Nilsson, M. and Simsarin, K.T., Cooperative Multi-Robot Box-Pushing, Proceedings of the 1995 IEEE/RSJ International Conference on Intelligent Robots and Systems (IROS' 95), Pittsburgh, PA, USA, (1995), pp. 556-561.

(11) Mataric, M.J., Designing and Understanding
Adaptive Group Behavior, Adaptive Behavior, Vol. 4, No. 1 (1995), pp. 51-80.

(12) Murata, S., Kurokawa, H. and Kokaji, S., SelfAssembling Machine, Proceedings of the 1994 IEEE International Conference on Robotics and Automation (ICRA '94), San Diego, CA, USA, (1994), pp. 441-448.

(13) Orwig, T., Cybermotion's Roving Robots, Industrial Robot, Vol. 20, No. 3 (1993), pp. 27-29.

(14) Yamaguchi, H., Adaptive Formation Control for Distributed Autonomous Mobile Robot Groups, Proceedings of the 1997 IEEE International Conference on Robotics and Automation (ICRA '97), Albuquerque, NM, USA, (1997), pp. 2300-2305.

(15) Yamaguchi, H., A Cooperative Hunting Behavior by Mobile Robot Troops, Proceedings of the 1998 IEEE International Conference on Robotics and Automation (ICRA '98), Leuven, Belgium, (1998), pp. 3204-3209.

(16) Yamaguchi, H. and Burdick, J.W., Asymptotic Stabilization of Multiple Nonholonomic Mobile Robots Forming Group Formations, Proceedings of the 1998 IEEE International Conference on Robotics and Automation (ICRA '98), Leuven, Belgium, (1998), pp. 3573-3580.

(17) Yamaguchi, H., A Cooperative Hunting Behavior by Mobile Robot Troops, the International Journal of Robotics Research, Vol.18, No.8 (1999), pp. 931-940. 